\title{
Magnetic Color-flavor-locked Stars
}

\author{
Laura Paulucci $^{1}$, Efrain J. Ferrer ${ }^{2}$, Vivian de la Incera ${ }^{2}$ \\ and Jorge E. Horvath ${ }^{3}$ \\ ${ }^{1}$ Universidade Federal do ABC, Brazil \\ email: laura.paulucci@ufabc.edu.br \\ ${ }^{2}$ University of Texas at El Paso, USA \\ ${ }^{2}$ Universidade de São Paulo, Brazil
}

\begin{abstract}
Using the solutions of the gap equations of the magnetic-color-flavor-locked (MCFL) phase of paired quark matter in a magnetic field, and taking into consideration the separation between the longitudinal and transverse pressures due to the field-induced breaking of the spatial rotational symmetry, the equation of state (EoS) of the MCFL phase is self-consistently determined. Implications for stellar models of magnetized (self-bound) strange stars and hybrid (MCFL core) stars are discussed.
\end{abstract}

Keywords. stars: neutron, magnetic fields, dense matter, equation of state

\section{Introduction}

Determining the state of nuclear matter in the interior of neutron stars is still an open question. It has been proposed that these stars are not composed of neutron matter, but rather that, given the conditions of very high density in their interiors, there could be a phase transition from nuclear to quark matter (e.g., Bodmer (1971), Witten (1984), Alcock et al. (1986), Glendenning \& Weber (1992)).

About a decade ago, when paired matter was studied (e.g., Alford et al. (1999), Rapp et al. (2000), Alford et al. (2001), Lugones \& Horvath (2002)), the pairing energy was shown to enlarge the window of stability in parameter space. The most symmetric pairing state at sufficiently high densities would be the Color-Flavor-Locked one (CFL) when quarks of all flavors and colors pair.

Many neutron stars present a strong magnetization. Their surface magnetic fields range from $\mathrm{B}=1.7 \times 10^{8} \mathrm{G}$ up to $2.1 \times 10^{13} \mathrm{G}$, with a typical value of $10^{12} \mathrm{G}$. Magnetars present evidences for even stronger magnetic fields - with surface magnetic fields of order $\mathrm{B} \sim 10^{14-15} \mathrm{G}$. By applying the equipartition theorem, the interior field can be estimated to reach values $\sim 10^{19-20} \mathrm{G}$ (Ferrer et al. 2010).

In this proceeding, we report some effects of a magnetic field in the EoS of the CFL phase as well as their implications for stellar models.

\section{Equation of State}

The CFL superconductor can be modeled by the three-flavor Nambu-Jona-Lasinio (NJL) theory and we assume a uniform and constant magnetic field, the MCFL phase (Ferrer et al. 2005, 2006). We can write the MCFL thermodynamic potential as the sum of the contributions coming from charged (C) and neutral (N) quarks, $\Omega_{M C F L}=\Omega_{C}+\Omega_{N}$, please refer to Paulucci et al. (2011) and references therein for more details.

The effects of confinement, as well as the pure magnetic energy contribution, are incorporated by including in $\Omega_{M C F L}$ a bag constant $B$ and the term $\tilde{H}^{2} / 2$, respectively: 
$\Omega_{H}=\Omega_{M C F L}+B+\tilde{H}^{2} / 2$, where $\tilde{H}$ is the rotated magnetic field (Alford et al. 1999) and the gaps are determined from their respective gap equations. The values of the free parameter $\mathrm{G}$ are chosen to produce a $\mathrm{CFL}$ gap of $10 \mathrm{MeV}$ at zero magnetic field, $\mathrm{G}=$ $4.32 \mathrm{GeV}^{-2}$, or $25 \mathrm{MeV}$ for $\mathrm{G}=5.15 \mathrm{GeV}^{-2}$ at $\mu=500 \mathrm{MeV}$.

The pressure and energy density of the MCFL phase are given by:

$$
\begin{gathered}
\epsilon_{M C F L}=\Omega_{H}-\mu \frac{\partial \Omega_{H}}{\partial \mu}, \\
p_{M C F L}^{\|}=-\Omega_{H}, \\
p_{M C F L}^{\perp}=-\Omega_{H}+\widetilde{H} \frac{\partial \Omega_{H}}{\partial \widetilde{H}}
\end{gathered}
$$

The conditions for absolute stability are that both parallel and perpendicular pressures are zero simultaneously. If we were to consider that the vacuum energy B does not depend on the magnetic field, in order to have absolute stability we would need the magnetization of the system to equal the magnetic field itself, which is not feasible. So for absolute stability, it is necessary to have a field-dependent bag constant (Paulucci et al. 2011).

For gravitationally bound stars, the own gravitational field can supply the pressure to compensate the internal one produced by the magnetic field. For such systems, keeping $\mathrm{B}$ constant in the EoS is in principle possible. Under this assumption we considered a fixed B-value in Fig. 1.

The EoS is largely linear and substantially modified only at sufficiently high fields where the magnetized medium becomes highly anisotropic. An interesting feature is that the EoS is not made substantially harder within this model with the increase in the value of the gap parameter, represented by the change in the value of $\mathrm{G}$. It is in contrast with the approach used in Alford et al. (2001) and Lugones \& Horvath (2002), for example, in which a higher $\Delta$ stiffens the EoS, rendering a higher maximum mass for strange stars. It is necessary to understand this difference in modeling color superconducting quark matter since it will certainly affect the way we analyze mass-radius relations.
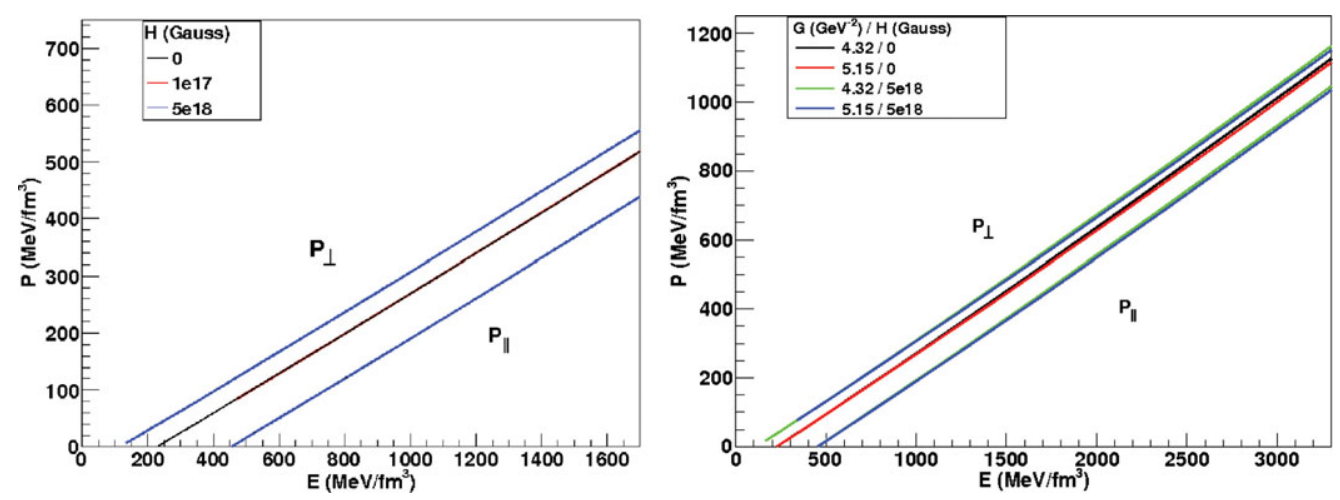

Figure 1. Left panel: EoS for MCFL matter considering parallel and perpendicular pressures for different values of $\mathrm{H}$, as indicated, $\mathrm{B}=58 \mathrm{MeV} / \mathrm{fm}^{3}$ and $\mathrm{G}=5.15 \mathrm{GeV}^{-2}$. Right panel: Comparison between the EoS for CFL matter for $\mathrm{H}=0$ and $\mathrm{H}=5 \times 10^{18} \mathrm{G}$ and $\mathrm{G}=4.32$ $\mathrm{GeV}^{-2}$ and $\mathrm{G}=5.15 \mathrm{GeV}^{-2}$ as indicated. 

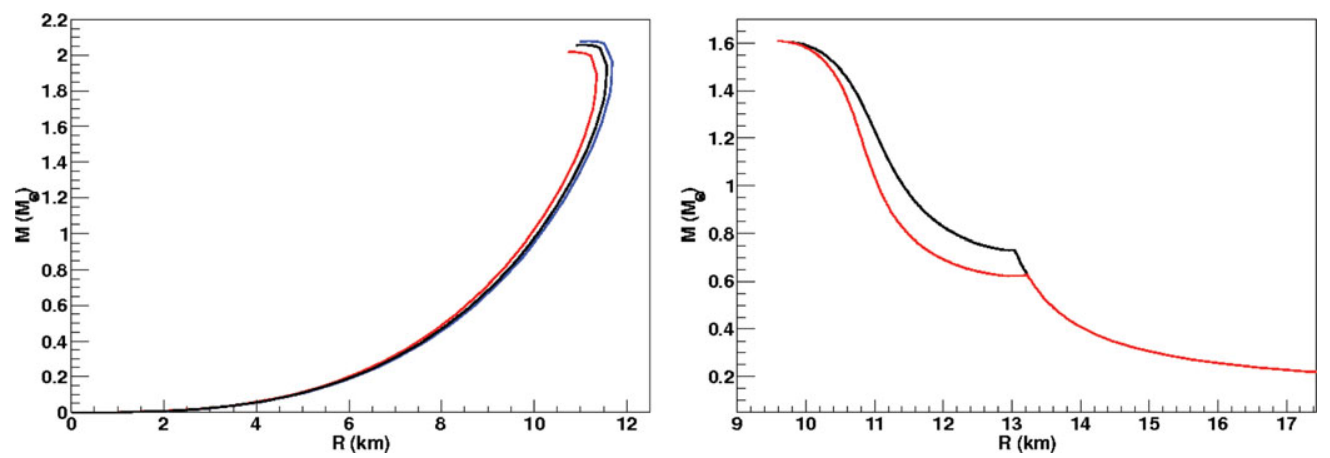

Figure 2. Mass-radius relation for magnetized CFL stars with $\mathrm{G}=5.15 \mathrm{GeV}^{-2}$. On the left panel: MCFL strange stars for $\mathrm{B}=58 \mathrm{MeV} / \mathrm{fm}^{3}$. The black line is for $\mathrm{H}=0$, whereas the red and blue lines are for the parallel and perpendicular pressures at $\mathrm{H}=1 \times 10^{18} \mathrm{G}$, respectively. On the right panel: hybrid stars with MCFL cores and employing the zero-temperature Bethe-Johnson EoS for nuclear matter, $\mathrm{B}=100 \mathrm{MeV} / \mathrm{fm}^{3}$, and using the perpendicular pressure. The black line is for $\mathrm{H}=0$ and the red one for $\mathrm{H}=1 \times 10^{18} \mathrm{G}$.

\section{Stellar Models}

The TOV equations apply to isotropic EoS, while the previous results indicate a rapidly growing anisotropy of the EoS beyond a certain field. The perpendicular pressure provides a harder EOS whereas the parallel is softer. In this way the use of the symmetric TOV equations is restricted to weak magnetic fields (i.e. smaller than $\sim 10^{18} \mathrm{G}$ ), when the deviation from spherical symmetry is very small $(<10 \%)$.

In Fig. 2 we present, as an example, the MR relation for when the anisotropy is still small. To work in the anisotropic regime an entirely different stellar structure formalism in agreement with the system cylindrical symmetry would be needed.

The recent detection of a $1.97 \pm 0.04 M_{\odot}$ pulsar by Demorest et al. (2010) imposes restrictions in parameter space for the EoS of both nuclear and quark matter. This result is consistent with strange MCFL stars with low magnetic fields but do not seem consistent with hybrid MCFL stars. We intend to analyze hybrid sequences employing a non-linear Walecka model with mean field approximation to see how the maximum mass is modified by the parameters in the theory.

\section{Acknowledgements}

The authors wish to acknowledge the financial support received from FAPESP, CNPq Agency (Brazil) and the Office of Nuclear Theory of the Department of Energy under contract de-sc0002179.

\section{References}

C. Alcock, E. Farhi \& A. V. Olinto, Astrophys. J. 310, 261 (1986)

M. Alford, K. Rajagopal \& F. Wilczek, Nuc. Phys. B537, 433 (1999)

M. Alford, et al., Phys. Rev. D 64, 074017 (2001)

A. R. Bodmer, Phys. Rev. D 4, 1601 (1971)

P. B. Demorest, et al., Nature 467, 1081 (2010)

E. J. Ferrer, et al., Phys. Rev. Lett. 95, 152002 (2005); Nucl. Phys. B 74788 (2006)

E. J. Ferrer, et al., Phys. Rev. C 82, 065802 (2010)

N. K. Glendenning \& F. Weber, Astrophys. J. 400, 647 (1992)

G. Lugones \& J. E. Horvath, Phys. Rev. D 66, 074017 (2002)

L. Paulucci, et al., Phys. Rev. D 83, 043009 (2011)

R. Rapp, et al., Ann. Phys. (NY) 280, 35 (2000)

E. Witten, Phys. Rev. D 30, 272 (1984) 